RESEARCH

\title{
ACUTE CORONARY SYNDROME IN GERIATRIC PATIENTS IN AN INTENSIVE CARE UNIT
}

Turkish Journal of Geriatrics

DOI: 10.31086/tigeri.2021.227

2021; 24(3): 297-302

\section{- Suzan KESKIN ${ }^{1}$}

- Arzu Neslihan AKGÜN ${ }^{1}$

- Orçun ÇiFTÇi ${ }^{1}$

- Ibrahim Haldun MÜDERRISOĞLU

CORRESPONDANCE

\section{${ }^{1}$ Suzan KESKIN}

Başkent University Ankara Education and Training Hospital, Cardiology, Ankara, Turkey

\section{Phone: +905399502736}

e-mail: suzankeskin@yahoo.com

Received: May 25, 2021

Accepted: Aug 30, 2021

${ }^{1}$ Başkent University Ankara Education and Training Hospital, Cardiology, Ankara, Turkey

\section{Abstract}

Objective: The advancing age of acute coronary syndrome and the ageing population are leading to an increase in the number of elderly patients with acute coronary syndrome in our clinical practice.

In our study, we aimed to investigate the effect of acute coronary syndrome in the geriatric patient group.

Materials and Method: We retrospectively included geriatric patients who were in intensive care units because of different diagnoses, who also showed an acute coronary syndrome, and who had been diagnosed using the sequential organ failure assessment score. This score is used to describe the condition of a patient with sepsis and the extent of organ damage during treatment in an intensive care unit. We reviewed patients who were at Baskent University Faculty of Medicine between 25 March 2015 and 12 March 2020.

Results: We included 63 patients aged $77.27 \pm 7.65$ years. There were 40 (63.5\%) males and 23 (36.5\%) females. A total of 42 (89.4\%) patients died in the first 5 months, one $(2.1 \%)$ died between the 6th and 10th months, two (4.3\%) between the 11th and 20th months, and two (4.3\%) between the 21st and 30th months. We found a significant relationship between the sequential organ failure assessment score and mortality rate $(p<0.05)$. The sequential organ failure assessment score was reliable in predicting mortality in geriatric patients with acute coronary syndrome, with $57 \%$ sensitivity and $75 \%$ specificity.

Conclusion: Mortality of geriatric patients with acute coronary syndrome can be significantly determined using the sequential organ failure assessment scores.

Keywords: Geriatrics; Acute coronary syndrome; Intensive Care Units; Mortality. 


\section{INTRODUCTION}

A simple but effective method for describing organ dysfunction or organ failure in critically ill patients is the sequential organ failure assessment (SOFA) score. The SOFA score was designed to provide insights into the acute morbidity of intensive care patients at the population level, but its use has expanded considerably in recent years. The SOFA score is now used at the individual patient level as a key criterion for diagnosing sepsis syndrome (14). Due to good medical treatment, the age of the population is increasing, which implies that acute coronary syndrome is now more common in geriatric patients (5).

Despite improvements in patient care and advanced treatment modalities, mortality remains a significant problem in geriatric patients. Elderly patients have a high risk of bleeding $(6,7)$ and ischemic complications (8), among other conditions. For this reason, careful risk stratification for ischemic risk and bleeding risk must be carried out, taking into account the assessment of frailty, quality of life, care goals, and individual preferences (9). With the help of the SOFA score, we aimed to identify the survival of elderly patients with acute coronary syndrome who were treated in the intensive care unit of our clinic.

\section{MATERIALS AND METHOD}

We retrospectively included geriatric patients who were in the intensive care unit because of different diagnoses and who also showed an acute coronary syndrome at Baskent University Faculty of Medicine between 25 March 2015 and 12 March 2020.

We analysed the demographic, clinical and biochemical data of these patients. We also applied the SOFA score to the patients. The sequential organ failure assessment score (SOFA score) is used to monitor a person's condition or failure rate to determine the extent of organ function during a person's stay in the intensive care unit (ICU). The score is based on six different scores, each for the respiratory, cardiovascular, liver, coagulation, kidney, and neurological systems. The SOFA score helps healthcare providers estimate the risk of morbidity and mortality from sepsis.

\section{Statistics}

We examined various demographic and clinical factors, including age, sex, age, coronary artery disease, hyperlipidaemia, diabetes, hypertension, and this was followed by a ROC curve and Fisher's Exact Test analysis to determine the independent predictors of mortality.

This study was approved by Baskent University Institutional Review Board (Project no: KA21/23) and supported by Baskent University Research Fund.

\section{RESULTS}

We included 63 patients aged $77.27 \pm 7.65$ years. There were 40 (63.5\%) males and 23 (36.5\%) females. The demographic and clinical properties of the study population are presented in Table 1. A total of $47(75.0 \%)$ patients died at a mean follow-up duration of 3.0 \pm 7.0 (range 0-30) months. Thirty-two (68.1\%) of these patients died in the hospital, while 15 (31.9\%) died outside the hospital.

In the first 5 months, 42 (89.4\%) patients died, whereas one $(2.1 \%)$ patient died in the 6th to 10 th month (2.1\%), two (4.3\%) between the 11th and 20th month, and two (4.3\%) between the 21th and 30th months.

The cut-off value was determined as 6.5 in the diagnosis of individuals with low and high SOFA. Based on this, we examined patients in two groups (SOFA low and SOFA high). For the cut-off value of SOFA of 6.5 , the sensitivity value was 0.76 .

In our study, we found a significant relationship between SOFA score and mortality rate $(p<0.05)$. The SOFA score was reliable for predicting mortality in geriatric patients with acute coronary syndrome, with $57 \%$ sensitivity and $75 \%$ specificity. 
Table 1. Demographic and clinical properties of the study population

\begin{tabular}{|l|c|}
\hline \multicolumn{1}{|c|}{ Characteristic } & Results \\
\hline Sex (Male,\%) & $63.5 \%$ \\
Age (years, mean \pm SD) & $77.27 \pm 7.65$ \\
Time to death (month, mean \pm SD) & $3.0 \pm 7.0$ mean \pm SD \\
Death (\%) & \\
- Total \% & $47(75 \%)$ \\
- In hospital \% & $15(31.9 \%)$ \\
- Outside the hospital \% & $28(44.4 \%)$ \\
Coronary artery disease (n,\%) & $45(71.4 \%)$ \\
HT (n,\%) & $21(33.3 \%)$ \\
HL (n,\%) & $22(34.9 \%)$ \\
DM (n,\%) & $8(12.7 \%)$ \\
Smoker (n,\%) & \\
\hline
\end{tabular}

We also found that there was no significant relationship between SOFA score and troponin values ( $p>0.05$ ). ROC analysis was performed to compare the diagnosing predictability of SOFA score and troponin values obtained as metric. According to $\mathrm{ROC}$ analysis, the usefulness of troponin values in distinguishing between individuals with low and high SOFA was not found to be significant (area under the curve $=0.529 p=0.710$ ), as shown in Figure 2.

Sensitivity value was set as the rate of accurately diagnosing individuals in the low-SOFA group based on troponin measurement. Similarly, the specificity value was set as the rate at which individuals in the high-SOFA group can be correctly diagnosed. The cut-off value of troponin values in the diagnosis of individuals with low and high SOFA was 1.30. At this cut-off value, the sensitivity of troponin was 0.71 . Thus, the diagnosing rate of individuals
Figure 1. The SOFA score sensitivity and specificity.

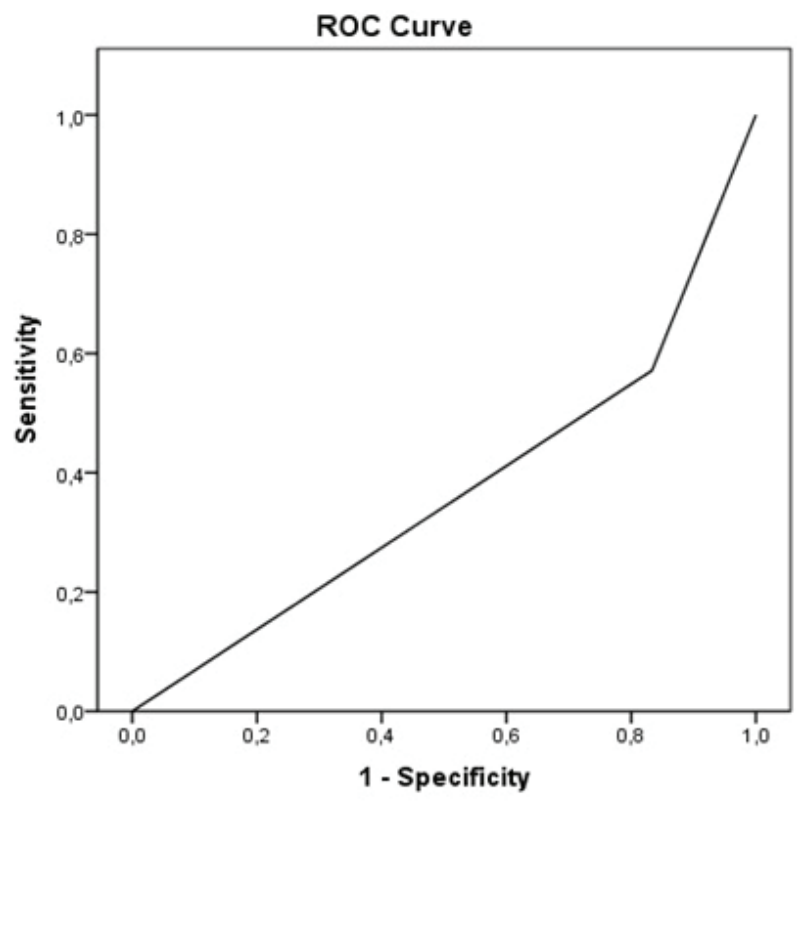

Figure 2. Roc analysis of troponin success

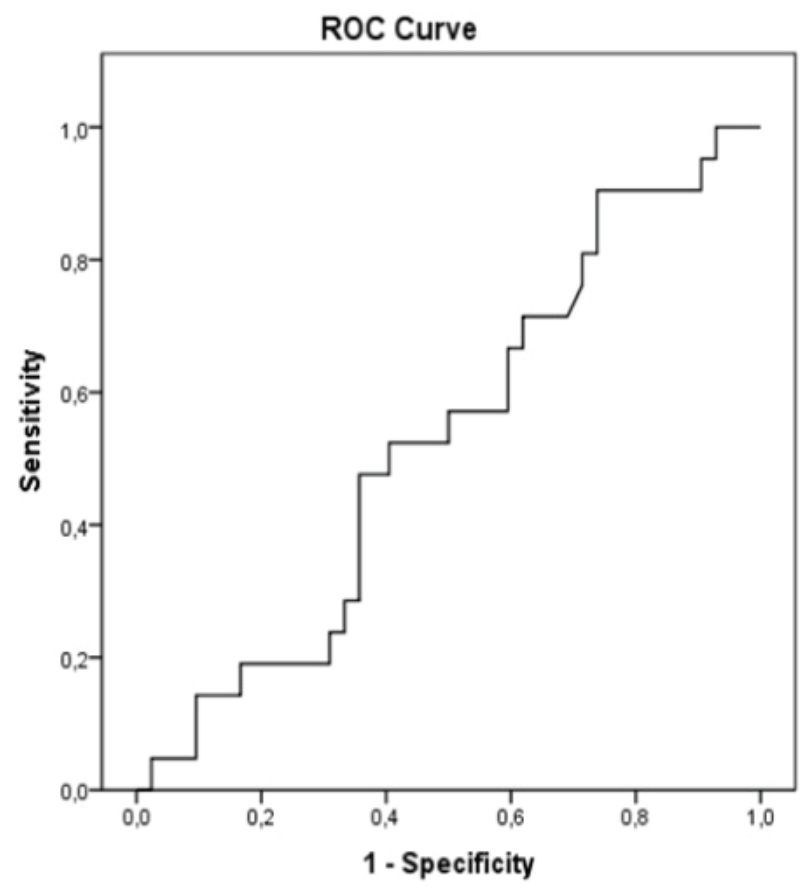


whose troponin measurement was lower than 1.30 was $71 \%$.

Further, we found no significant relationship between SOFA scores and CK-MB values ( $p>0.05)$. In this case, the sensitivity value was set as the rate of correctly diagnosing individuals in the low-SOFA based on CK-MB measurement. The specificity value was set as the rate at which individuals in the high-SOFA group could be correctly diagnosed. The cut-off value of CK-MB values was determined as 2.45 in the diagnosis of individuals with low and high SOFA. At this cut-off value, the sensitivity of CK-MB was 0.71 . This indicates that the rate of diagnosing individuals whose CKMB value was lower than 2.45, who were diagnosed as SOFA-low, was $71 \%$.

\section{DISCUSSION}

Geriatrics refers to the medical care of older adults, who are patients older than 65 years and have chronic illnesses, physical impairments and/or cognitive impairments. With the high quality of current medical treatment, the mean age of geriatric patients has increased, with concurrent increases in the age of patients with acute coronary syndrome. For this reason, the treatment of very old patients has steadily increased $(10,11)$ and has become common practice.

Due to changes and the physiology of ageing, comorbidities, and expectations increase in the elderly. Geriatric patients must be assessed individually, as there can be significant differences between their chronological and biological ages (12). In this context, the SOFA score can be used to assess life expectancy. A simple but effective method for describing organ dysfunction or organ defects for critically ill patients (13).

In our study, we found a significant relationship between SOFA score and mortality rate $(p<0.05)$. The SOFA score showed that it is reliable for predicting mortality in geriatric patients with acute coronary syndrome, with $57 \%$ sensitivity and $75 \%$ specificity.

Jones et al. (14) also showed that the SOFA score provides valuable prognostic information on survival in hospital. Machado et al. (15) showed that a mean SOFA average above 5 was associated with mortality in elderly patients with severe sepsis and septic shock. Janssens et al. (16) showed that SOFA scores provide important information on the degree and progression of organ dysfunction in medical and cardiovascular patients.

ACS refers to a range of acute ischemic myocardial conditions. It includes unstable angina and myocardial infarction with or without ST segment elevation. ACS can lead to an increase in cardiac enzymes (17), with an increase in the values of troponin and CK-MB. As part of our study, we carefully examined the relationship between the troponin value and SOFA score and between the CK-MB value and SOFA score. We found that there was no significant relationship between SOFA score and troponin values or CK-MB values $(p>0.05)$.

However, Mannam et al. (18) showed that the mortality in troponin-positive septic patients was significantly higher $(45.4 \%$ versus $7.7 \%, p<0.04$ ). Kang et al. (19) showed that elevated troponin levels were significantly associated with short-term and long-term mortality in patients with end-stage kidney disease with sepsis. For this reason, elevated troponin levels should be carefully considered and monitored for undesirable results. Oliveira et al. (20) showed that the severity of septic disease was the only variable that was significantly associated with death. Mehta et al. (21) showed that cardiac troponin is an independent predictor of hospital mortality.

Nevertheless, a skilful clinical assessment remains essential in every patient with suspected ACS and is particularly important in elderly patients who present with specific diagnostic challenges that need to be systematically considered. 


\section{CONCLUSION}

The mortality of geriatric patients with acute coronary syndrome can be significantly determined by their SOFA scores. The SOFA scores showed reliability in predicting mortality in geriatric patients with acute coronary syndrome, with $57 \%$ sensitivity and $75 \%$ specificity. The SOFA score thus provides potentially valuable prognostic information on the survival of geriatric patients in the hospital. It is therefore very well suited for the risk stratification of patients in intensive care units.

\section{REFERENCES}

1. Lambden S, Laterre PF, Levy MM, Francois B. The SOFA score development, utility and challenges of accurate assessment in clinical trials. Crit Care 2019; 27;23(1):374 (PMID: 31775846).

2. Seymour CW, Liu VX, Iwashyna TJ, et al. Assessment of Clinical Criteria for Sepsis: For the Third International Consensus Definitions for Sepsis and Septic Shock (Sepsis-3). JAMA. 2016;23;315(8):762-74 (PMID: 26903335).

3. Shankar-Hari M, Phillips GS, Levy ML, et al. Developing a New Definition and Assessing New Clinical Criteria for Septic Shock: For the Third International Consensus Definitions for Sepsis and septic Shock (Sepsis-3). JAMA. 2016;315(8):775-787 (PMID: 26903336)

4. Singer M, Deutschman CS, Seymour $C$, et al. The Third International Consensus Definitions for Sepsis and Septic Shock (Sepsis-3). JAMA. 2016;315(8):801810 (PMID: 26903338).

5. Çimci M, Karadağ B, Acute coronary syndrome in the elderly. Turk Kardiyol Dern Ars 2017;45 (Suppl 5): 35-38. (PMID: 28976379).

6. Lip Gregory Y.H, Clementy N, Pericart L, Banerjee A, Fauchier L, Stroke and major bleeding risk in elderly patients aged $\geq 75$ years with atrial fibrillation: The Loire Valley atrial fibrillation project. Stroke. 2015;46(1):143-150. (PMID: 25424474).

7. Riobóo-Lestón L, Raposeiras-Roubin S, Abu-Assi E, Iñiguez-Romo A. Bleeding risk assessment in elderly patients with acute coronary syndrome. J Geriatr Cardiol. 2019;16(2): 145-150 (PMID: 30923546).

\section{Study Limitations}

The limitations of our study are its retrospective design and the relatively low number of patients.

In addition, patients who were not followed up in our hospital were not included in our study.

\section{Conflict of Interest}

The authors declare no conflict of interest regarding the publication of this manuscript.

8. Crimi G, Morici N, Ferrario M, et al. Time Course of Ischemic and Bleeding Burden in Elderly Patients with Acute Coronary Syndromes Randomized to Low-Dose Prasugrel or Clopidogrel. J Am Heart Assoc. 2019; 22;8(2) (PMID: 30636561).

9. Engberding N, Wenger NK, Acute Coronary Syndroms in the Elderly. Journal List. 2017;6: 1791 (PMID: 29043079).

10. Mayhew LD. Health and Elderly Care Expenditure in an Aging World. IIASA Research Report. IIASA, Laxenburg, Austria 2000, pp 42-43.

11. Ihra GC, Lehberger J, Hochrieser H, et al. Development of demographics and outcome of very old critically ill patients admitted to intensive care units. Intensive Care Med. 2012;38(4): 620-626 (PMID: 22354500).

12. Padilla IM, Martin-Asenjo R, Bueno H. Management of Acute Coronary Syndromes in Geriatric Patients. Heart Lung Circ. 2017;26 (2): 107-113 (PMID: 27641094).

13. Qiao Q, Lu G, Li M, Shen Y, Xu D. Prediction of Outcome in Critically III Elderly Patients using APACHE II and SOFA Scores. J Int Med Res 2012;40(3):11141121. (PMID: 22906285).

14. Jones AE, Trzeciak S, Kline JA. The Sequential Organ Failure Assessment score for predicting outcome in patients with severe sepsis and evidence of hypoperfusion at the time of emergency department presentation. Crit Care Med. 2009;37(5):1649-1654. (PMID: 19325482).

15. Machado RL, David CM, Oliveira GMM, et al. Association of the SOFA score and mortality in elderly pa- 
tients with severe sepsis and septic shock. Crit Care. 2005;9(Suppl 2): P49. (DOI:10.1186/cc3593).

16. Janssens U, Graf C, Graf J, et al. Evaluation of the SOFA score: a single-center experience of a medical intensive care unit in 303 consecutive patients with predominantly cardiovascular disorders. Sequential Organ Failure Assessment. Intensive Care Med. 2000;26(8):1037-1045. (PMID: 11030159).

17. James SK, Armstrong P, Barnathan E, et al. and GUSTO-IV-ACS-Investigators. Troponin and C-reactive protein have different relations to subsequent mortality and myocardial infarction after acute coronary syndrome: A GUSTO-IV substudy. J Am Coll Cardiol. 2003;41(6) 916-924 (PMID: 12651034).

18. Mannam P, Devarakonda VS, Wittbrodt ET, et al. Association of troponin I concentrations with outcomes in sepsis. CHEST Journal. 2004; 86(5S): 126. Poster presentations. (DOI:10.1378/chest.126.4_MeetingAbstracts.865S).

19. Kang EW, Na HJ, Hong SM, et al. Prognostic value of elevated cardiac troponin I in ESRD patients with sepsis Nephrol. Dial. Transplant 2009; 24 (5):15681573 (PMID: 19145004).

20. Oliveira NS, Silva VR, Castelo JS, et al. Serum level of cardiac troponin I in pediatric patients with sepsis or septic shock. Pediatr Crit Care Med. 2008; 9(4): 414417 (PMID: 18843251).

21. Mehta NJ, Khan IA, Gupta V, et al. Cardiac troponin I predicts myocardial dysfunction and adverse outcome in septic shock. Int. J. Cardiol 2004; 95(1): 1317(PMID: 15159032). 